\title{
Molecular Plant Pathology
}

\section{Targeting Fusarium graminearum control via polyamine enzyme inhibitors and polyamine analogues}

\begin{tabular}{|r|l|}
\hline Journal: & Molecular Plant Pathology \\
\hline Manuscript ID: & Draft \\
\hline Manuscript Type: & Original Article \\
\hline Date Submitted by the Author: & n/a \\
\hline Complete List of Authors: & $\begin{array}{l}\text { Crespo-Sempere, Ana; University of Lleida, Applied Mycology Unit, Food } \\
\text { Technology Department } \\
\text { Estiarte, Núria; University of Lleida, Applied Mycology Unit, Food } \\
\text { Technology Department } \\
\text { Marín, Sonia; University of Lleida, Applied Mycology Unit, Food Technology } \\
\text { Department } \\
\text { Sanchis, Vicente; University of Lleida, Applied Mycology Unit, Food } \\
\text { Technology Department } \\
\text { Ramos, Antonio Javier; University of Lleida, Applied Mycology Unit, Food } \\
\text { Technology Department }\end{array}$ \\
\hline Keywords: & $\begin{array}{l}\text { <i>Fusarium graminearum</i>, FHB, deoxynivalenol, wheat, polyamines, } \\
\text { DFMo }\end{array}$ \\
\hline
\end{tabular}


1 Targeting Fusarium graminearum control via polyamine enzyme

2 inhibitors and polyamine analogues

3

4 Crespo-Sempere, A., Estiarte, N., Marín, S., Sanchis, V., Ramos, A.J.

5

6 Applied Mycology Unit, Food Technology Department. University of Lleida. UTPV-

$7 \quad$ XaRTA, Agrotecnio Center. Av. Rovira Roure 191. 25198 Lleida. Spain.

8 ana.crespo@udl.cat

9

10 Abstract

11 Fusarium graminearum has major impact in wheat cultivation as it not only reduces

12 yield and seed quality but also constitutes a risk to public or animal health owing to its

13 ability to contaminate grains with mycotoxins. Under high inoculum conditions,

14 chemical fungicides are the main measure to control F. graminearum. However,

15 resistance problems are emerging and control strategies based on new targets are

16 needed. Polyamines are low molecular weight cations which have a key role in growth,

17 development and differentiation. In this work, the possibility of using polyamine

18 metabolism as a target to control $F$. graminearum has been assessed. It was found that

19 the polyamine putrescine induces mycotoxin production, correlating with an over

20 expression of TRI5 and TRI6 genes that are involved in deoxynivalenol (DON)

21 biosynthesis and regulation, respectively. In addition, a homolog of the Saccharomyces

22 cerevisiae TPO4, which is involved in the excretion of putrescine, was up-regulated as

23 the concentration of putrescine in the medium increased while DUR3 and SAM3

24 homologues, involved in putrescine uptake, were down-regulated. When $2.5 \mathrm{mM} \mathrm{D}$, L-

$25 \alpha$-difluoromethylornithine (DFMO), an inhibitor of the enzyme ornithine decarboxylase 
26 that catalyzes the first and committed step in the polyamine synthesis, was added to the

27 medium, DON production decreased from 3.2 to $0.06 \mathrm{ng} / \mathrm{mm}^{2}$ of colony, while the 28 growth was lowered by up to 70 per cent. Interestingly, an over expression of the 29 ornithine decarboxilase gene was observed when the $F$. graminearum culture was 30 amended with DFMO. However, exogenous putrescine could overcome the effects of 31 DFMO on growth and mycotoxin production. Because polyamines are regulated by its 32 biosynthesis, degradation and transport, five polyamine transport inhibitors were also 33 tested against $F$. graminearum. AMXT-1505 was able to completely inhibit in vitro 34 both growth and DON production. When wheat spikes were treated with DFMO and 35 subsequently inoculated with $F$. graminearum conidia, DFMO did not display 36 inhibitory effects on the fungus. Nevertheless, AMXT-1505 blocked F. graminearum 37 growth in inoculated wheat spikes, reducing DON mycotoxin contamination from 76.87 $38 \mu \mathrm{g} / \mathrm{g}$ present at the positive control to $0.62 \mu \mathrm{g} / \mathrm{g}$. Therefore, the polyamine transport 39 inhibitor AMXT-1505 may be useful as a target specific fungicide against $F$. 40 graminearum.

Keywords: Fusarium graminearum; FHB; deoxynivalenol; wheat; polyamines; DFMO

\section{Introduction}

Fusarium graminearum is one of the most important causal agents of the fungal

46 disease Fusarium Head Blight (FHB) of wheat and other small-grain cereals that not 47 only reduces yields but also reduces seed quality and contaminates grains with mycotoxins (Parry et al., 1995; Stępień and Chełkowski, 2010). The disease occurs throughout much of the world and often with a devastating impact on wheat crops

50 (Goswami and Kistler, 2004; McMullen et al., 1997). The most common mycotoxins 
51 associated with $F$. graminearum are deoxynivalenol (DON), nivalenol (NIV) and 52 zearalenone (ZEA) which pose a health risk to humans and animals through food and 53 feed with accumulated mycotoxins (D'Mello et al., 1999; Desjardins and Proctor, 54 2007). However, DON is known to be the most commonly detected mycotoxin in cereal 55 grains, usually at high concentrations (Canady et al., 2001). At a cellular level, DON 56 can inhibit protein synthesis by binding to the ribosome and activating cellular protein 57 kinases involved in signal transduction related to proliferation, differentiation, and 58 apoptosis (Pestka and Smolinski, 2005). In mammals, DON can cause emetic effects, 59 anorexia and immune dysregulation as well as growth, reproductive and teratogenic 60 effects (Pestka, 2010).

61 To control fungal contamination, several management strategies have been 62 employed, which include FHB-resistant wheat cultivars obtained by breeding processes 63 or through transgenic approaches, chemical fungicides, biological control and cultural 64 practices (Blandino et al., 2012; Yuen and Schoneweis, 2007). However, under high 65 inoculum conditions, chemical fungicides are the main measure to control the impact of 66 the $F$. graminearum infection. Among the fungicides reported to be effective against 67 FHB, triazols are the most used, which are sterol biosynthesis inhibitors (Paul et al., 68 2010). Nevertheless, since fungi are able to evolve and adapt, fungicide-resistant strains 69 may arise. Indeed, a decrease of triazol fungicide efficiency has been found (Becher et 70 al., 2010; Klix et al., 2007; Yin et al., 2009). Additionally, several authors have 71 reported that sub-lethal doses of some of these fungicides may increase mycotoxin 72 production from $F$. graminearum (D'Mello et al., 1998; Müllenborn et al., 2008; Ochiai 73 et al., 2007). Consequently, there is a clear need to design control strategies based on 74 new targets. 
Over the past few years a number of studies have analyzed the role of

76 polyamines (PA) in fungal metabolism, which has been recently reviewed by Valdés-

77 Santiago et al. (2012). Polyamines are low molecular weight aliphatic polycations with

78 two or more primary amino groups, ubiquitously present in all living cells. PA are

79 involved in growth, development and differentiation, being its concentration highly

80 regulated. Main PA in fungi are putrescine, spermidine, and spermine, although some

81 fungal species do not produce spermine. Interestingly, it has been observed that the PA

82 biosynthetic pathway is induced in the wheat as an early response to FHB. Moreover, it

83 has been reported that intermediates of the PA pathway promote DON biosynthesis

84 (Gardiner et al., 2010; Gardiner et al., 2009). In this study we have considered PA

85 metabolism as a target to design new control strategies (Figure 1). To this aim, we

86 studied the effect of putrescine and D, L- $\alpha$-difluoromethylornithine (DFMO), an

87 inhibitor of the enzyme ornithine decarboxylase which catalyzes the first and committed

88 step in the synthesis of PA (Metcalf et al., 1978), on growth, DON production and

89 infection capacity. Additionally, we investigated the possibility of controlling $F$.

90 graminearum with lipophilic polyamine analogues, produced by $\mathrm{N}$-acylation or $\mathrm{N}$ -

91 alkylation of the $\varepsilon$-amine group of the lysine portion of the Lys-spermine conjugates,

92 which can contribute to the inhibition of polyamine transport. As PA transport has a key

93 role in the regulation of PA content in cell, we also analyzed the gene expression of six

94 proteins putatively involved in PA transport.

95

96 2. Results and discussion

$97 \quad 2.1$ Influence of putrescine on $F$. graminearum

98 To examine the effect of exogenous putrescine on $F$. graminearum, the fungus

99 was grown on plates with increasing amounts of putrescine $(0,50,500,1000$ and 5000 
$\mu \mathrm{M})$. As Figure 2A indicates, DON increases proportionally to putrescine concentration,

101 resulting in almost a 23 fold induction when plates were amended with $5000 \mu \mathrm{M}$ of

102 putrescine. These results are similar to those reported previously by Gardiner et al.

103 (2009) who conducted a large-scale nutrient profiling to assess the role that different

104 carbon and nitrogen sources play in the production of DON. These authors found that

105 DON production was strongly induced in liquid culture by some amine compounds

106 which included putrescine. Thus, it was confirmed that putrescine has a key role on

107 mycotoxin production. In contrast to DON production, the growth of $F$. graminearum

108 was not affected by the putrescine concentrations assayed. However, it would appear

109 that the effect of putrescine on growth depends on the fungal species as Rajam and

110 Galston (1985) found that the addition of putrescine to the culture medium resulted in a

111 promotion of growth in Botrytis cinerea, Monilia fructicola and Rhizoctonia solani.

112 Additionally, Figure 2B shows the colonies of the $F$. graminearum strain

113 incubated with increasing amounts of putrescine, which suggests that other metabolites

114 as carotenoids may also be influenced by putrescine due to the variation of colony color

115 among putrescine concentrations. Indeed, Rodríguez-Ortiz et al. (2009) showed

116 regulatory connections between nitrogen metabolism and carotenogenesis and

117 secondary metabolism in Fusarium fujikuroi.

118 To further elucidate the influence of putrescine on $F$. graminearum, a gene 119 expression analysis was carried out (Figure 3). We studied TRI5 and TRI6 genes 120 involved in DON biosynthesis and regulation (Hohn et al., 1993; Proctor et al., 1995), 121 the ornithine decarboxylase $(O D C)$ and polyamine oxidase $(P A O)$ which are involved in 122 PA biosynthesis and oxidation, respectively, and TPO1, TPO4, TPO5, UGA4, DUR3 123 and $S A M 3$, identified by Valdes et al. (2012) by homology through an in silico search of 124 well-characterized PA transport proteins of Saccharomyces cerevisiae. Figure 3 reveals 
125 that the increment of DON production observed in Figure 2A was correlated with an 126 overexpression of TRI5 and TRI6 genes, showing at $5000 \mu \mathrm{M}$ of putrescine a $\log _{2}$ Ratio 127 of 11.5 and 8.2 for TRI5 and TRI6, respectively, which represent a 2896.3 and 294.1128 fold change. Consequently, it appears that putrescine can modulate DON biosynthesis 129 affecting the transcriptional regulator encoded by TRI6 and the trichodiene synthase 130 TRI5, which catalyzes the first step in DON biosynthesis. It should be noted that TRI5 131 was found to be positively regulated by TRI6 (Proctor et al., 1995). These results concur 132 with the findings of Gardiner et al. (2009) who observed that putrescine induced the 133 fluorescence of a $F$. graminearum strain that contained a GFP gene regulated by the 134 TRI5 promoter.

135 By contrast, it was found that the addition of exogenous putrescine to a $F$. 136 graminearum culture had no effect on the $O D C$ and $P A O$ expression. Because $O D C$ 137 catalyzes the first and committed step in the synthesis of PA, it seems that the 138 concentration of putrescine is not self-regulatory at a transcriptional level in $F$. 139 graminearum. Concerning to the gene expression of the putative PA transporters 140 analyzed in this study, we found an up-regulation of TPO4 and a down-regulation of 141 DUR3 and SAM3 when cultures were amended with increasing putrescine 142 concentrations (Figure 3). Transport of PA in fungi has been poorly investigated and the 143 existing studies are mainly focused in S. cerevisiae (Igarashi and Kashiwagi, 2010; 144 Valdés-Santiago et al., 2012). In S. cerevisiae, TPO1 and TPO4, located mainly in the 145 plasma membrane, are the major PA excretion proteins (Tomitori et al., 2001; Tomitori 146 et al., 1999; Uemura et al., 2005). Nevertheless, TPO5 which is located on Golgi or 147 post-Golgi secretory vesicles and UGA4, located on the vacuolar membrane, can also 148 catalyze the excretion of PA (Tachihara et al., 2005; Uemura et al., 2004). Furthermore, 149 DUR3 and SAM3 are the major PA uptake proteins in yeasts (Uemura et al., 2007). 
150 Assuming that these proteins may have similar functions in $F$. graminearum, the 151 increase of putrescine in the culture media would lead to an overexpression of TPO4 to 152 raise the excretion of putrescine, and a downregulation of DUR3 and SAM3 to reduce 153 the putrescine uptake.

154

155

\subsection{Inhibition of putrescine biosynthesis in F. graminearum by DFMO}

To reduce PA biosynthesis, we tested the DFMO capacity to inhibit ODC, which catalyze the first step in the synthesis of PA (Figure 1). When $2.5 \mathrm{mM}$ of DFMO was added to the culture media, growth and DON production were greatly reduced as shown in Figure 4. DON production decreased from 3.2 to $0.06 \mathrm{ng} / \mathrm{mm}^{2}$ of colony while the growth was lowered by up to 70 per cent. However, when the culture media was supplemented with $1 \mathrm{mM}$ of putrescine, the DON production and the growth were recovered. Similar behavior was observed when Penicillium citrinum, Aspergillus parasiticus and Aspergillus nidulans where treated with PA inhibitors, as mycelial growth and citrinin, aflatoxin and sterigmatocystin production, respectively, were reduced by PA inhibitors (Giridhar et al., 1997; Guzmán-de-Peña et al., 1998; Guzmánde-Peña and Ruiz-Herrera, 1997). Additionally, PA inhibitors were able to in vitro inhibit the growth of other phytopathogenic fungi such as B. cinerea, R. solani, Cochliobolus carbonum, Colletotrichum truncatum, Fusarium oxysporium and Phytophtora infestans (Gamarnik et al., 1994; Rajam and Galston, 1985; Walters, 1995).

As putrescine increased TRI5 and TRI6 expression and DON production (Figure 3), it might be expected that DFMO, which binds to ODC decreasing its activity and consequently the putrescine biosynthesis, will reduce TRI5 and TRI6 expression. However, TRI5 and TRI6 gene expression was up-regulated when DFMO was added to 
175 the media (Figure 5). Supplementation of $1 \mathrm{mM}$ of exogenous putrescine together with $1762.5 \mathrm{mM}$ of DFMO resulted in an increase of TRI5 and TRI6 gene expression at similar 177 levels to those found when $F$. graminearum cultures were amended with just $1 \mathrm{mM}$ of 178 putrescine (Figure 3 and 5). Therefore, there must be a DON regulation system 179 downstream of putrescine production. Another interesting finding was the up-regulation 180 of $O D C$ when $F$. graminearum cultures were treated with DFMO to inhibit the ODC 181 activity, showing a $\log _{2}$ Ratio of 4.8 which represents a 27.9 -fold change. With respect 182 to PA transporters, F. graminearum showed an up-regulation of TPO1, TPO4 and $183 U G A 4$ as well as a down-regulation of DUR3 in response to DFMO. Based on these 184 results, it would seem that DFMO induces PA excretion proteins and transport to the 185 vacuolar membrane and represses PA uptake proteins but further analysis needs to be 186 performed. inhibit the cellular PA uptake. To investigate whether the PA transport could be inhibited in F. graminearum, five different PA analogs, kindly provided by Aminex

\subsection{Inhibition of PA transport in $F$. graminearum}

Although DFMO inhibits largely DON production, the fungi may import PA from the wheat during the infection process, overcoming the inhibition of putrescine biosynthesis. Intracellular PA concentrations are regulated by not only biosynthesis and degradation but also transport. Therefore, PA transport may be a useful target to control F. graminearum infection and DON contamination. For some years now, the inhibition of PA transport has been a goal for medical research as elevated levels of PA have been associated with different types of cancer (Nowotarski et al., 2013). In this context, Burn et al. $(2001 ; 2009)$ developed a group of lipophilic polyamine analogues that potently Therapeutics (USA), were tested. To this aim, F. graminearum was inoculated in plates 
200 amended with $1 \mathrm{mM}$ of putrescine as this could be a common PA concentration during 201 plant infection. Results are presented as relative with respect to the control (1 mM 202 putrescine) (Figure 6). The observed effect on growth and DON production largely 203 depends on the Polyamine Transport Inhibitor (PTI) used. While AMXT-1483 reduced 204 growth and increased DON production by as much as $180 \%$, AMXT-1505 reduced 205 completely both DON production and mycelial growth when $600 \mu \mathrm{M}$ of this PTI was 206 added to the medium. AMXT-2030 had little effect on DON production and mycelial 207 growth and AMXT-3016 increased DON production by $270 \%$ but did not affect to the 208 growth. In addition, AMXT-3946 reduced DON production up to $70 \%$ and increased 209 the mycelial growth up to $210 \%$. Of them all, we should highlight the AMXT-1505 210 PTI, as it was able to efficiently control F. graminearum. Additionally, when AMXT2111505 was applied at sub-lethal doses, not only it does not increase DON production as 212 observed for other fungicides but reduces it, minimizing the risk of mycotoxin 213 contamination (D'Mello et al., 1998; Müllenborn et al., 2008; Ochiai et al., 2007).

214 To further investigate the possible mode of action of AMXT-1505, gene 215 expression analysis of genes involved in DON biosynthesis and regulation, PA 216 biosynthesis and oxidation and PA transport was carried out in cultures amended with 217100,300 and $500 \mu \mathrm{M}$ of this PTI (Figure 7). However, little changes were observed 218 with respect to the control, showing a concentration dependent down-regulation of 219 TPO1 and to a lesser extent TPO5. In the case that AMXT-1505 was really able to 220 inhibit PA transport, it may lead to PA accumulation inside the cell which in turn may 221 cause cytotoxicity. In fact, excessive level of PA may be coupled to the induction of 222 apoptosis (Schipper et al., 2000). Nonetheless, it is difficult to speculate a mode of 223 action for AMXT-1505 and more research is needed. 


\subsection{Antifungal activity of DFMO and AMXT-1505 against $F$. graminearum in} wheat

To verify whether DFMO and AMXT-1505 are able to control $F$. graminearum growth and mycotoxin production in wheat, spikes of wheat were treated with these compounds to be later point inoculated by placing a droplet of conidia suspension in the middle of the spikes. The conditions upon which inoculated spikes were incubated were highly favorable for the $F$. graminearum growth as the inoculation was performed in spikes harvested at anthesis and incubated at $26^{\circ} \mathrm{C}$ and $100 \% \mathrm{RH}$ for 12 days. Firstly, it should be pointed out that spikes were free from external contamination as shown by the negative control in the Figure 8. Additionally, it should be noted that $F$. graminearum grew in all the spikes from the positive control, forming a white mycelium that covered almost the entire length of the spike. Interestingly, although DFMO reduced the fungal growth in vitro by $70 \%$, when spikes were pre-treated with DFMO, F. graminearum was able to grow even more than in the positive control. Conversely, AMXT-1505 was capable of controlling F. graminearum with only light growth in two of the spikes. As for the DON production, a concentration of $76.87 \mu \mathrm{g} / \mathrm{g}$ was found in the positive control while pre-treated spikes with DFMO and AMXT-1505 contained 212 and $0.62 \mu \mathrm{g} / \mathrm{g}$, respectively. From the results obtained, noteworthy is the need of validating the results obtained in vitro as DFMO switched from a $98 \%$ reduction to a $276 \%$ increase of DON production. These findings may be explained considering the possibility that the fungus overcomes the inhibition of putrescine biosynthesis importing the putrescine from the wheat. Bharti and Rajam (1995) found that some DFMO treatments caused an increase of PA levels in wheat that may be responsible for the DON increase observed in Figure 8 since higher putrescine concentrations produce higher amounts of DON as shown in Figure 2. 
Although this kind of PTIs have not been tested before as fungicides against $F$.

251 graminearum, some researchers have reported other PA analogues and conjugates used

252 to control phytopathogenic fungi. The putrescine analogue 1-aminooxy-3-aminopropane

253 did not reduce mycelial growth of the phytopathogenic fungus Sclerotinia sclerotiorum,

254 but perturbed polyamine metabolism inhibiting the enzyme ornithine decarboxylase,

255 spermidine synthase, and S -adenosyl-methionine decarboxilase in mycelial extracts

256 (Gárriz et al., 2003). In addition, it was found that two spermidine analogues, N,N-

257 dimethyl-N1-(3-aminopropyl)-1,3-diaminopropane trihydrochloride and N,N-dimethyl-

258 N1-(3-aminopropyl)-1,4-diaminobutane trihydrochloride, reduced growth of the fungal

259 plant pathogen Pyrenophora avenae (Mackintosh et al., 2001). Walters et al. (2001)

260 found PA conjugates, isolated from pollen of Quercus alba, which have antifungal

261 activity against $P$. avenae and Mellon and Moreau (2004) showed a combination of two

262 polyamine conjugates that reduced aflatoxin production but had no effect on growth of

263 Aspergillus flavus.

264 The results reported in this study indicate that the use of PA analogs is a 265 promising approach for the control of the FHD and DON contamination. Since there is a 266 need to find new targets to overcome resistance problems, AMXT-1055 may be useful 267 as a target specific fungicide against $F$. graminearum, contributing to limit crop losses 268 and to reduce the risk of contamination by mycotoxins in food and feed.

270 3. Material and methods

$271 \quad 3.1$ Strain and growth conditions

272 The F. graminearum strain used in this study was L1-2/3D, kindly provided by

273 Dr. Belén Patiño from the Department of Microbiology III, Faculty of Biology, 274 University Complutense of Madrid (Spain). This strain was isolated from Spanish wheat 
275 and identified by PCR using Fg16F and Fg16R primers (Nicholson et al., 1998). To

276 enable production of macroconidia, the isolate was sub-cultured on plates containing, 277 per liter, 30 g sucrose, $2 \mathrm{~g} \mathrm{NaNO}_{3}, 1 \mathrm{~g} \mathrm{KH}_{2} \mathrm{PO}_{4}, 0.5 \mathrm{~g} \mathrm{MgSO}_{4} \cdot 7 \mathrm{H}_{2} \mathrm{O}, 0.5 \mathrm{~g} \mathrm{KCl}$ and 100 $278 \mu \mathrm{L}$ of trace element solution (per $100 \mathrm{~mL}, 5 \mathrm{~g}$ citric acid, $5 \mathrm{~g} \mathrm{ZnSO} \cdot 7 \mathrm{H}_{2} \mathrm{O}, 0.25 \mathrm{~g}$

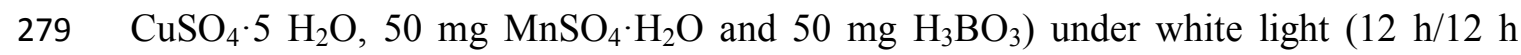
280 light/dark) at $26{ }^{\circ} \mathrm{C}$ for 6 days. Conidia were collected with a sterile solution of $0.005 \%$ $281(\mathrm{v} / \mathrm{v})$ Tween 80 , and adjusted to $10^{5}$ conidia/mL using a Thoma counting chamber. 282 Plates with the media mentioned above but containing 1 gram of glutamine instead of 2 $283 \mathrm{~g} \mathrm{NaNO}_{3}$, were inoculated centrally with $5 \mu \mathrm{L}$ of conidia suspensions to study the effect 284 of putrescine (SIGMA), DFMO (Enzo Life Sciencies) and five polyamine transport 285 inhibitors (PTI), detailed in Table 1, kindly provided by Aminex Therapeutics (USA). 286 Putrescine concentrations assayed were 50, 500, 1000 and $5000 \mu \mathrm{M}$, the DFMO 287 concentration tested was $2.5 \mathrm{mM}$, while the PTIs concentrations analyzed ranged from 28850 to $1000 \mu \mathrm{M}$. Putrescine, DFMO and the PTIs were dissolved in water, sterilized by 289 passing the solution through a $0.45 \mu \mathrm{m}$ pore size filter and added to the autoclaved 290 culture media. For growth assessment, two perpendicular diameters of the growing 291 colonies were measured after incubation for 3 days under white light $(12 \mathrm{~h} / 12 \mathrm{~h}$ 292 light/dark) at $26^{\circ} \mathrm{C}$.

\subsection{Extraction and detection of DON from culture}

To extract DON, one agar plug ( $7 \mathrm{~mm}$ in diameter) was removed from the center 296 of the colonies incubated for 7 days under white light $(12 \mathrm{~h} / 12 \mathrm{~h} \mathrm{light} /$ dark $)$ at $26{ }^{\circ} \mathrm{C}$.

297 The agar plug was shaken for $60 \mathrm{~s}$ in $750 \mu \mathrm{L}$ of water and left for $60 \mathrm{~min}$ at room 298 temperature. Then, vials were shaken and the extracts were filtered (Millex-HV 0.45 $299 \mu \mathrm{m}, 25 \mathrm{~mm}$, Millipore Corporation) into another vial. DON extracts were stored at -20 
$300{ }^{\circ} \mathrm{C}$ until quantification. DON was measured using the RIDASCREEN ${ }^{\circledR}$ DON enzyme

301 immunoassay test from R-BIOPHARM, according to the manufacturer's instructions.

302

303

3.3. Gene expression analysis

304 Mycelia were collected from 7 days old cultures grown on media amended with 305 putrescine, DFMO or PTIs. The mycelium was frozen in liquid nitrogen and stored at $306-80{ }^{\circ} \mathrm{C}$ before nucleic acid extraction. RNA was extracted from the mycelium ground to 307 a fine powder with a mortar and pestle using liquid nitrogen. Powdered mycelium was 308 added to a pre-heated $\left(65^{\circ} \mathrm{C}\right)$ mixture of $10 \mathrm{~mL}$ of extraction buffer $(100 \mathrm{mM}$ Tris$309 \mathrm{HCl}, \mathrm{pH}$ 8.0, $100 \mathrm{mM} \mathrm{LiCl}, 10 \mathrm{mM}$ EDTA, 1\% (w/v) sodium dodecyl sulfate, 1\% (w/v) 310 polyvinyl-pyrrolidone $40,1 \%(\mathrm{v} / \mathrm{v}) \beta$-mercaptoethanol) and $5 \mathrm{~mL}$ of Tris-equilibrated 311 phenol. The extract was incubated at $65^{\circ} \mathrm{C}$ for $15 \mathrm{~min}$ and cooled before adding $5 \mathrm{~mL}$ 312 of chloroform:isoamyl alcohol $(24: 1, \mathrm{v} / \mathrm{v})$. The homogenate was centrifuged at $3900 \times \mathrm{g}$ 313 for $20 \mathrm{~min}$ at $4{ }^{\circ} \mathrm{C}$, and the aqueous phase was re-extracted with $10 \mathrm{~mL}$ of 314 phenol:chloroform:isoamyl alcohol (25:24:1, v/v/v). RNA was precipitated during $3 \mathrm{~h}$ at $315-20{ }^{\circ} \mathrm{C}$ by adding $3.3 \mathrm{~mL}$ of $12 \mathrm{M} \mathrm{LiCl}$. After centrifugation at $27,200 \times \mathrm{g}$ for $60 \mathrm{~min}$, 316 the pellet was washed with $500 \mu \mathrm{L}$ of $70 \%$ ethanol. The resulting pellet was re317 extracted with $250 \mu \mathrm{L}$ of $3 \mathrm{M}$ sodium acetate (pH 6.0) to remove residual 318 polysaccharides. Then, RNA was washed again with $500 \mu \mathrm{L}$ of $70 \%$ ethanol, and, 319 finally, dissolved in $200 \mu \mathrm{L}$ of water. RNA concentration was measured 320 spectrophotometrically and verified by agarose gel electrophoresis $(1.2 \%)$ and 321 ethidium-bromide staining. Total RNA was treated with DNase (TURBO DNase, 322 Ambion) to remove contaminating genomic DNA. Single-strand cDNA was synthesized 323 from $5 \mu \mathrm{g}$ of total RNA using SuperScript III reverse transcription kit and an oligo(dT) 324 primer, according to the manufacturer's instructions (Invitrogen). 
APT5newF and APT5newR were used as gene-specific primer set to amplify the

326

327

328

329

330

331

332

333

334

335

336

337

338

339

340

341

342

343

344

345

346

347

348

349

TRI5 gene (Mudge et al., 2006). The rest of the primer sets were designed with OLIGO

Primer Analysis Software V.7 (Table 2). Real-time RT-PCR reactions were performed on a CFX96 Touch ${ }^{\mathrm{TM}}$ Real-Time PCR Detection System (BIO-RAD) monitoring cDNA amplification with SsoAdvanced ${ }^{\mathrm{TM}}$ SYBR ${ }^{\circledR}$ Green Supermix (BIO-RAD). The primer pair BETA-6_F and BETA-6_R was designed within the beta tubulin gene for using it as a reference gene. The standard protocol included one cycle at $98{ }^{\circ} \mathrm{C}$ for $2 \mathrm{~min}$, followed by 40 cycles at $98{ }^{\circ} \mathrm{C}$ for $5 \mathrm{~s}$ and $56^{\circ} \mathrm{C}$ for $30 \mathrm{~s}$. The corresponding real-time PCR efficiency (E) in the exponential phase was calculated according to the equation: $\mathrm{E}$ $=10[-1 /$ slope $]$. The relative expression of the target genes was calculated based on the $\mathrm{E}$ and the Crossing point $(\mathrm{Cp})$ value of the sample versus the control. The $\mathrm{Cp}$ value is the cycle at which fluorescence from amplification exceeds the background fluorescence. The relative expression of the target genes was expressed in comparison to the beta tubulin gene (reference gene), according to the following equation (Pfaffl, 2001; Rasmussen, 2001): ratio $=\left(\mathrm{E}_{\text {target }}\right)^{\Delta \mathrm{Cp} \text { target(control - sample })} /\left(\mathrm{E}_{\mathrm{ref}}\right)^{\Delta \mathrm{Cp} \text { ref(control - sample })}$. Gene expression measures were derived from biological triplicates.

\subsection{Plant growth and inoculation}

Wheat plants (Triticum aestivum), cultivar Califa sur, were grown in a greenhouse with temperatures that ranged from 14 to $32^{\circ} \mathrm{C}$ during the day and 7 to $12^{\circ} \mathrm{C}$ at night. Spikes of wheat were harvested at or shortly before anthesis. Spikes were surface-sterilized by dipping them into a $\mathrm{NaClO}$ solution $(0.1 \% \mathrm{Cl})$ for $15 \mathrm{~min}$, followed by $2 \mathrm{~min}$ in ethanol (70 \%). Excess of ethanol was removed by placing spikes in a laminar flow bench. The DFMO and AMXT-1505 PTI treatments were performed by dipping the spikes into the solutions for 2 minutes and placing the spikes in a laminar flow bench until they were 
350 dried (about 10 minutes), after which spikes were inoculated. Four different treatments

351 were tested consisting of spikes point inoculated in the middle of the spike with $10 \mu \mathrm{L}$

352 of conidial suspension $\left(10^{5}\right.$ conidia/mL) or water, as positive and negative controls, and 353 spikes treated with $2.5 \mathrm{mM}$ DFMO or $700 \mu \mathrm{M}$ AMXT-1505 inoculated with $10 \mu \mathrm{L}$ of 354 conidial suspension. Transparent plastic boxes $300 \mathrm{~mm}$ x $160 \mathrm{~mm}$ x $180 \mathrm{~mm}$ deep were 355 filled with a beaker with water to give a RH of $100 \%$, a data logger (Escort iLog RH) 356 recording temperature and $\mathrm{RH}$ at 30 min intervals and a rack where spikes were placed 357 vertically separated from one each other. Boxes were kept in an incubator at $26^{\circ} \mathrm{C}$ with $35812 \mathrm{~h} / 12 \mathrm{~h}$ light/dark photoperiod for 12 days. A box with 10 spikes was used for each 359 treatment.

360

\section{Acknowledgements}

362 This research has been supported by the Spanish Government (AGL2011-24862 363 project). N. Estiarte thanks the Comissionat per a Universitats i Recerca, del 364 Departament d'Innovació, Universitats i Empresa de la Generalitat de Catalunya 365 (AGAUR), for her predoctoral grant.

366 4. References

367 Becher, R., Hettwer, U., Karlovsky, P., Deising, H. B. and Wirsel, S. G. R. (2010) 368 Adaptation of Fusarium graminearum to tebuconazole yielded descendants 369 diverging for levels of fitness, fungicide resistance, virulence, and mycotoxin $370 \quad$ production. Phytopathology 100, 444-453.

371 Bharti and Rajam, M. V. (1995) Effects of the polyamine biosynthesis inhibitor 372 difluoromethylornithine on growth, polyamine levels, chromosome behaviour 373 and polygenic traits in wheat (Triticum aestivum L.). Ann. Bot-London 76, $297-$ 374 301. 
375 Blandino, M., Haidukowski, M., Pascale, M., Plizzari, L., Scudellari, D. and Reyneri, 376 A. (2012) Integrated strategies for the control of Fusarium head blight and deoxynivalenol contamination in winter wheat. Field Crops Res. 133, 139-149.

Burns, M. R., Carlson, C. L., Vanderwerf, S. M., Ziemer, J. R., Weeks, R. S., Cai, F., Webb, H. K. and Graminski, G. F. (2001) Amino acid/spermine conjugates:

Burns, M. R., Graminski, G. F., Weeks, R. S., Chen, Y. and O'Brien, T. G. (2009) Lipophilic lysine--spermine conjugates are potent polyamine transport inhibitors for use in combination with a polyamine biosynthesis inhibitor. J. Med. Chem. 52, 1983-1993.

Canady, R., Coker, R., Rgan, S., Krska, R., Kuiper-Goodman, T., Olsen, M., Pestka, J., Resnik, S. and Schlatter, J. (2001) Deoxynivalenol. Safety evaluation of certain mycotoxins in food. Fifty-sixth report of the Joint FAO/WHO Expert Committee on Food Additives. WHO Food Additives Series, 47 International Programme on Chemical Safety-World Health Organization, Geneva, pp. 420-555.

D'Mello, F. J. P., Macdonald, A. C., Postel, D., Dijksma, W. P., Dujardin, A. and Placinta, C. (1998) Pesticide use and mycotoxin production in Fusarium and Aspergillus phytopathogens. Eur. J. Plant Pathol. 104, 741-751.

D’Mello, J. P. F., Placinta, C. M. and Macdonald, A. M. C. (1999) Fusarium mycotoxins: a review of global implications for animal health, welfare and productivity. Anim. Feed Sci. Technol. 80, 183-205.

Desjardins, A. E. and Proctor, R. H. (2007) Molecular biology of Fusarium mycotoxins. Int. J. Food Microbiol. 119, 47-50. 
399 Gamarnik, A., Frydman, R. B. and Barreto, D. (1994) Prevention of infection of 400 soybean seeds by Colletotrichum truncatum by polyamine biosynthesis

401

402

403

404

405

406

407

408

409

410

411

412

413

414

415

416

417

418

419

420

421

422

423 inhibitors. Phytopathology 84, 1445-1448.

Gardiner, D., Kazan, K., Praud, S., Torney, F., Rusu, A. and Manners, J. (2010) Early activation of wheat polyamine biosynthesis during Fusarium head blight implicates putrescine as an inducer of trichothecene mycotoxin production. BMC Plant Biol. 10, 289.

Gardiner, D. M., Kazan, K. and Manners, J. M. (2009) Nutrient profiling reveals potent inducers of trichothecene biosynthesis in Fusarium graminearum. Fungal Genet. Biol. 46, 604-613.

Gárriz, A., Dalmasso, M., Pieckenstain, F. and Ruiz, O. (2003) The putrescine analogue 1-aminooxy-3-aminopropane perturbs polyamine metabolism in the phytopathogenic fungus Sclerotinia sclerotiorum. Arch. Microbiol. 180, 169175.

Giridhar, P., Reddy, S. M. and Rajam, M. (1997) Control of Penicillium citrinum growth and citrinin production by some polyamine biosynthesis inhibitors. Indian Phytopathol. Soc. 50, 33-36.

Goswami, R. S. and Kistler, H. C. (2004) Heading for disaster: Fusarium graminearum on cereal crops. Mol. Plant Pathol. 5, 515-525.

Guzmán-de-Peña, D., Aguirre, J. and Ruiz-Herrera, J. (1998) Correlation between the regulation of sterigmatocystin biosynthesis and asexual and sexual sporulation in Emericella nidulans. A. Van Leeuw. 73, 199-205.

Guzmán-de-Peña, D. and Ruiz-Herrera, J. (1997) Relationship between aflatoxin biosynthesis and sporulation in Aspergillus parasiticus. Fungal Genet. Biol. 21, 198-205. 
424 Hohn, T., McCormick, S. and Desjardins, A. (1993) Evidence for a gene cluster 425 involving trichothecene-pathway biosynthetic genes in Fusarium 426 sporotrichioides. Curr. Genet. 24, 291-295.

427 Igarashi, K. and Kashiwagi, K. (2010) Characteristics of cellular polyamine transport in $428 \quad$ prokaryotes and eukaryotes. Plant Physiology and Biochemistry 48, 506-512.

429 Klix, M. B., Verreet, J.-A. and Beyer, M. (2007) Comparison of the declining triazole 430 sensitivity of Gibberella zeae and increased sensitivity achieved by advances in 431 triazole fungicide development. Crop Prot., 26, 683-690.

432 Mackintosh, C. A., Slater, L. A., Walters, D. R. and Robins, D. J. (2001) Synthesis of 433 six novel N,N-dialkyl derivatives of spermidine and effects on growth of the 434 fungal plant pathogen Pyrenophora avenae. FEMS Microbiol. Lett. 202, 221435225.

436 McMullen, M., Jones, R. and Gallenberg, D. (1997) Scab of wheat and barley: A re437 emerging disease of devastating impact. Plant Dis., 81, 1340-1348.

438 Mellon, J. E. and Moreau, R. A. (2004) Inhibition of aflatoxin biosynthesis in 439 Aspergillus flavus by diferuloylputrescine and p-coumaroylferuloylputrescine. $J$. $440 \quad$ Agric. Food Chem. 52, 6660-6663.

441 Metcalf, B. W., Bey, P., Danzin, C., Jung, M. J., Casara, P. and Vevert, J. P. (1978) 442 Catalytic irreversible inhibition of mammalian ornithine decarboxylase 443 (E.C.4.1.1.17) by substrate and product analogs. J. Am. Chem. Soc. 100, 25514442553.

445 Mudge, A. M., Dill-Macky, R., Dong, Y., Gardiner, D. M., White, R. G. and Manners, 446 J. M. (2006) A role for the mycotoxin deoxynivalenol in stem colonisation 447 during crown rot disease of wheat caused by Fusarium graminearum and 448 Fusarium pseudograminearum. Physiol. Mol. Plant Pathol. 69, 73-85. 
449 Müllenborn, C., Steiner, U., Ludwig, M. and Oerke, E.-C. (2008) Effect of fungicides 450 on the complex of Fusarium species and saprophytic fungi colonizing wheat $451 \quad$ kernels. Eur. J. Plant Pathol. 120, 157-166.

452 Nicholson, P., Simpson, D. R., Weston, G., Rezanoor, H. N., Lees, A. K., Parry, D. W. 453 and Joyce, D. (1998) Detection and quantification of Fusarium culmorum and 454 Fusarium graminearum in cereals using PCR assays. Physiol. Mol. Plant $455 \quad$ Pathol. 53, 17-37.

456 Nowotarski, S. L., Woster, P. M. and Casero, R. A. J. (2013) Polyamines and cancer: 457 implications for chemotherapy and chemoprevention. Expert Rev. Mol. Med. 15, 458 $28 \mathrm{pp}$.

Ochiai, N., Tokai, T., Takahashi-Ando, N., Fujimura, M. and Kimura, M. (2007) Genetically engineered Fusarium as a tool to evaluate the effects of environmental factors on initiation of trichothecene biosynthesis. FEMS Microbiol. Lett. 275, 53-61.

Parry, D. W., Jenkinson, P. and McLeod, L. (1995) Fusarium ear blight (scab) in small grain cereals - a review. Plant Pathology 44, 207-238.

Paul, P. A., McMullen, M. P., Hershman, D. E. and Madden, L. V. (2010) Metaanalysis of the effects of triazole-based fungicides on wheat yield and test weight as influenced by Fusarium Head Blight Intensity. Phytopathology 100, 160-171.

Pestka, J. (2010) Deoxynivalenol: mechanisms of action, human exposure, and toxicological relevance. Arch. Toxicol. 84, 663-679.

Pestka, J. J. and Smolinski, A. T. (2005) Deoxynivalenol: toxicology and potential effects on humans. J. Toxicol. Env. Health, Part B 8, 39-69. 
473 Pfaffl, M. W. (2001) A new mathematical model for relative quantification in real-time $474 \quad$ RT-PCR. Nucleic Acids Res. 29, E45.

475 Proctor, R. H., Hohn, T. M., McCormick, S. P. and Desjardins, A. E. (1995) Tri6 476 encodes an unusual zinc finger protein involved in regulation of trichothecene 477 biosynthesis in Fusarium sporotrichioides. Appl. Environ. Microbiol. 61, 1923$478 \quad 1930$.

479 Rajam, M. V. and Galston, A. W. (1985) The effects of some polyamine biosynthetic 480 inhibitors on growth and morphology of phytopathogenic fungi. Plant Cell $481 \quad$ Physiol. 26, 683-692.

482 Rasmussen, R. (2001) Quantification on the LightCycler instrument. In Meuer, S., 483 Wittwer, C. and Nakagawara, K. (eds), Rapid Cycle Real-time PCR: Methods 484 and Applications. Springer, Heidelberg, pp. 21-34.

485 Rodríguez-Ortiz, R., Limón, M. C. and Avalos, J. (2009) Regulation of carotenogenesis 486 and secondary metabolism by nitrogen in wild-type Fusarium fujikuroi and 487 carotenoid-overproducing mutants. Appl. Environ. Microbiol. 75, 405-413.

488 Schipper, R. G., Penning, L. C. and Verhofstad, A. A. J. (2000) Involvement of 489 polyamines in apoptosis. Facts and controversies: effectors or protectors? Semin. $490 \quad$ Cancer Biol. 10, 55-68.

491 Stępień, Ł. and Chełkowski, J. (2010) Fusarium head blight of wheat: pathogenic 492 species and their mycotoxins. World Mycotoxin J. 3, 107-119.

493 Tachihara, K., Uemura, T., Kashiwagi, K. and Igarashi, K. (2005) Excretion of 494 putrescine and spermidine by the protein encoded by YKL174c (TPO5) in 495 Saccharomyces cerevisiae. J. Biol. Chem. 280, 12637-12642. 
496 Tomitori, H., Kashiwagi, K., Asakawa, T., Kakinuma, Y., Michael, A. J. and Igarashi,

497 K. (2001) Multiple polyamine transport systems on the vacuolar membrane in 498 yeast. Biochem. J. 353, 681-688.

499 Tomitori, H., Kashiwagi, K., Sakata, K., Kakinuma, Y. and Igarashi, K. (1999)

500 Identification of a gene for a polyamine transport protein in yeast. J. Biol. Chem.

$501 \quad 274,3265-3267$.

502 Uemura, T., Kashiwagi, K. and Igarashi, K. (2007) Polyamine uptake by DUR3 and 503 SAM3 in Saccharomyces cerevisiae. J. Biol. Chem. 282, 7733-7741.

504 Uemura, T., Tachihara, K., Tomitori, H., Kashiwagi, K. and Igarashi, K. (2005)

505 Characteristics of the polyamine transporter TPO1 and regulation of its activity

506 and cellular localization by phosphorylation. J. Biol. Chem. 280, 9646-9652.

507 Uemura, T., Tomonari, Y., Kashiwagi, K. and Igarashi, K. (2004) Uptake of GABA and 508 putrescine by UGA4 on the vacuolar membrane in Saccharomyces cerevisiae. 509 Biochem. Biophys. Res. Commun. 315, 1082-1087.

510 Valdés-Santiago, L., Cervantes-Chávez, J. A., León-Ramírez, C. G. and Ruiz-Herrera, J. 511 (2012) Polyamine metabolism in fungi with emphasis on phytopathogenic $512 \quad$ species. J. Amino Acids 2012, 13.

513 Walters, D., Meurer-Grimes, B. and Rovira, I. (2001) Antifungal activity of three $514 \quad$ spermidine conjugates. FEMS Microbiol. Lett. 201, 255-258.

515 Walters, D. R. (1995) Inhibition of polyamine biosynthesis in fungi. Mycol. Res. 99, $516 \quad 129-139$.

517 Yin, Y., Liu, X., Li, B. and Ma, Z. (2009) Characterization of sterol demethylation 518 inhibitor-resistant isolates of Fusarium asiaticum and F. graminearum collected 519 from wheat in China. Phytopathology 99, 487-497. 
520 Yuen, G. Y. and Schoneweis, S. D. (2007) Strategies for managing Fusarium head 521 blight and deoxynivalenol accumulation in wheat. Int. J. Food Microbiol. 119, $522 \quad 126-130$.

523

524 Figure captions

525 Figure 1: Polyamines biosynthetic pathway.

526 Figure 2: A) Effect of putrescine on DON production (left y-axis) and relative colony 527 diameter (\%) referred to the control (right y-axis) (control $=100 \%$ ). B) Effect of 528 putrescine on $F$. graminearum colonies incubated for 7 days at $26{ }^{\circ} \mathrm{C}(12 \mathrm{~h} / 12 \mathrm{~h}$ 529 light/dark).

530 Figure 3: Relative expression of TRI5, TRI6, ODC, PAO, TPO1, TPO4, TPO5, UGA4, $531 D U R 3$ and $S A M 3$ in F. graminearum grown in the presence of different concentrations 532 of putrescine with respect to expression level in the same medium without putrescine. 533 Error bars indicate standard errors.

534 Figure 4: A) Effect of DFMO on DON production (left y-axis) and relative colony 535 diameter (\%) referred to the control (right y-axis) (control $=100 \%$ ). B) Effect of 536 DFMO on $F$. graminearum colonies incubated for 7 days at $26{ }^{\circ} \mathrm{C}(12 \mathrm{~h} / 12 \mathrm{~h}$ 537 light/dark).

538 Figure 5: Relative expression of TRI5, TRI6, ODC, PAO, TPO1, TPO4, TPO5, UGA4, $539 D U R 3$ and SAM3 in F. graminearum grown in the presence of DFMO and putrescine 540 with respect to expression level in the same medium without DFMO or putrescine. 541 Error bars indicate standard errors.

542 Figure 6: Effect of PTIs on DON production and colony diameter referred to the 543 control $(1000 \mu \mathrm{M}$ putrescine $)$ in percentage $($ control $=100 \%)$. 
544 Figure 7: Relative expression of TRI5, TRI6, ODC, PAO, TPO1, TPO4, TPO5, UGA4,

545 DUR3 and SAM3 in F. graminearum grown in the presence of $1000 \mu \mathrm{M}$ putrescine 546 different concentrations of AMTX-1505 with respect to expression level in the same 547 medium without AMTX-1505. Error bars indicate standard errors.

548 Figure 8: Activity of DFMO and AMXT-1505 against $F$. graminearum in wheat. 549 Spikes of wheat were harvested at or shortly before anthesis, point inoculated by 550 placing a droplet $(10 \mu \mathrm{L})$ of conidia suspension $\left(10^{5}\right.$ conidia $\left./ \mathrm{mL}\right)$ and incubated at $55126^{\circ} \mathrm{C}-100 \%$ RH with $12 \mathrm{~h} / 12 \mathrm{~h}$ light/dark photoperiod for 12 days.

552

553 Table legends:

554 Table 1: Polyamine transport inhibitors tested against $F$. graminearum.

555 Table 2: Primers used in this study. 


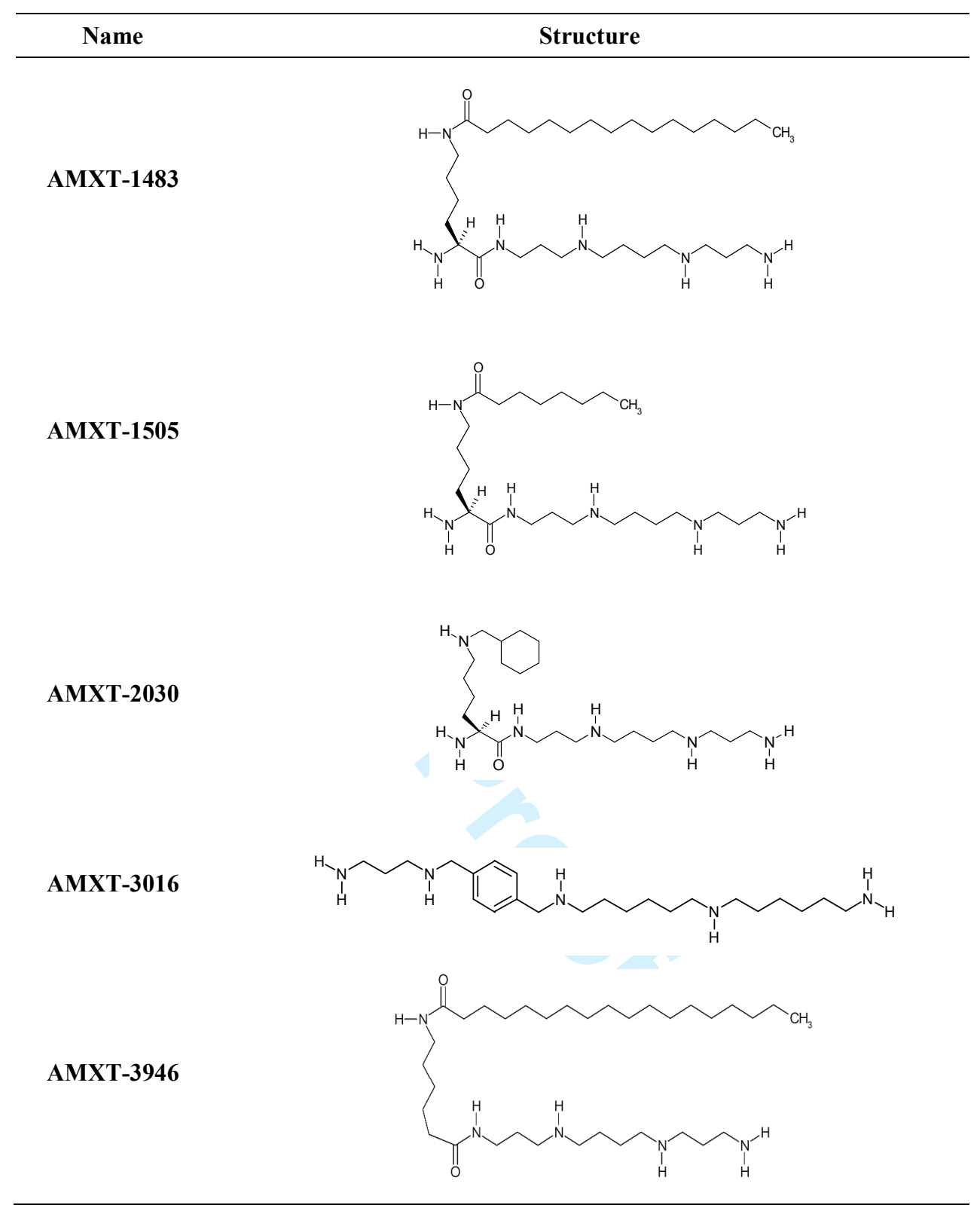

42

43

44

45

46

47

48

49

50

51

52

53

54

55

56

57

58

59

60 


\begin{tabular}{|c|c|c|c|}
\hline Gene number & Gene & Primer name & Primer sequence $\left(5^{\prime} \rightarrow 3^{\prime}\right)$ \\
\hline \multirow{2}{*}{ FGSG_03537 } & \multirow{2}{*}{ TRI5 } & APT5newF & CACTTTGCTCAGCCTCGCC \\
\hline & & APT5newR & CGATTGTTTGGAGGGAAGCC \\
\hline \multirow{2}{*}{ FGSG_16251 } & \multirow{2}{*}{ TRI6 } & TRI6_F & CACCTTCACACGGCCAAGCAA \\
\hline & & TRI6_R & ATGCCGCCTAAAGTCCCGTCC \\
\hline \multirow{2}{*}{ FGSG_05903 } & \multirow{2}{*}{$O D C^{*}$} & ODC_F & ACCGCATCACTGATAGCACTCG \\
\hline & & ODC_R & TGGTGTATGCGCCCATATCCTC \\
\hline \multirow{2}{*}{ FGSG_10284 } & \multirow{2}{*}{$P A O^{*}$} & PAO_F & ACTCTGAGAGCGATCCCGACT \\
\hline & & PAO_R & GCAGACCCTCCCGCATTGTCC \\
\hline \multirow{2}{*}{ FGSG_04370 } & \multirow{2}{*}{$T_{P O 1^{*}}$} & TOP1_F & GCTTGCCGCCAACAACGGTA \\
\hline & & TOP1_R & GGTGTATCCAGTCCATCCGAACCA \\
\hline \multirow{2}{*}{ FGSG_03725 } & \multirow{2}{*}{$\mathrm{TPO}^{*}$} & TOP4_F & CCATCTTTGCTGCCGCTCCC \\
\hline & & TOP4_R & CCATGACCCATCGCCAACCAG \\
\hline \multirow{2}{*}{ FGSG_02439 } & \multirow{2}{*}{ TPO5 $^{*}$} & TOP5_F & GCCACCATCTGTCTCGCCTCC \\
\hline & & TOP5_R & TCCACACGACGCAGATACCGTTG \\
\hline \multirow{2}{*}{ FGSG_06473 } & \multirow{2}{*}{$U G A 4^{*}$} & UGA4_F & GCCCTCCATGATCCCTGTTACCC \\
\hline & & UGA4_R & TGACCCCAGGCCCAGTACCAA \\
\hline \multirow{2}{*}{ FGSG_03111 } & \multirow{2}{*}{$D U R 3^{*}$} & DUR3_F & AGACTTATCGCCATCTCGCACAC \\
\hline & & DUR3_R & ACGCCCATCCAGACATATAGCC \\
\hline \multirow{2}{*}{ FGSG_00613 } & \multirow{2}{*}{$S A M 3^{*}$} & SAM3_F & ATATTCGCTTCCGCCGTGCCT \\
\hline & & SAM3_R & ATCCATGAGCCCACGACACCA \\
\hline \multirow{2}{*}{ FGSG_09530 } & \multirow{2}{*}{$\begin{array}{c}\text { BETA } \\
\text { TUBULIN }\end{array}$} & BETA-6_F & AGCTCACCCAGCAGATGTTCG \\
\hline & & BETA-6_R & GCGCATCTGGTCCTCAACCTCC \\
\hline
\end{tabular}

*Homologous genes identified by Valdes et al. (2012) by homology with an in silico search of well characterized Saccharomyces cerevisiae proteins. 


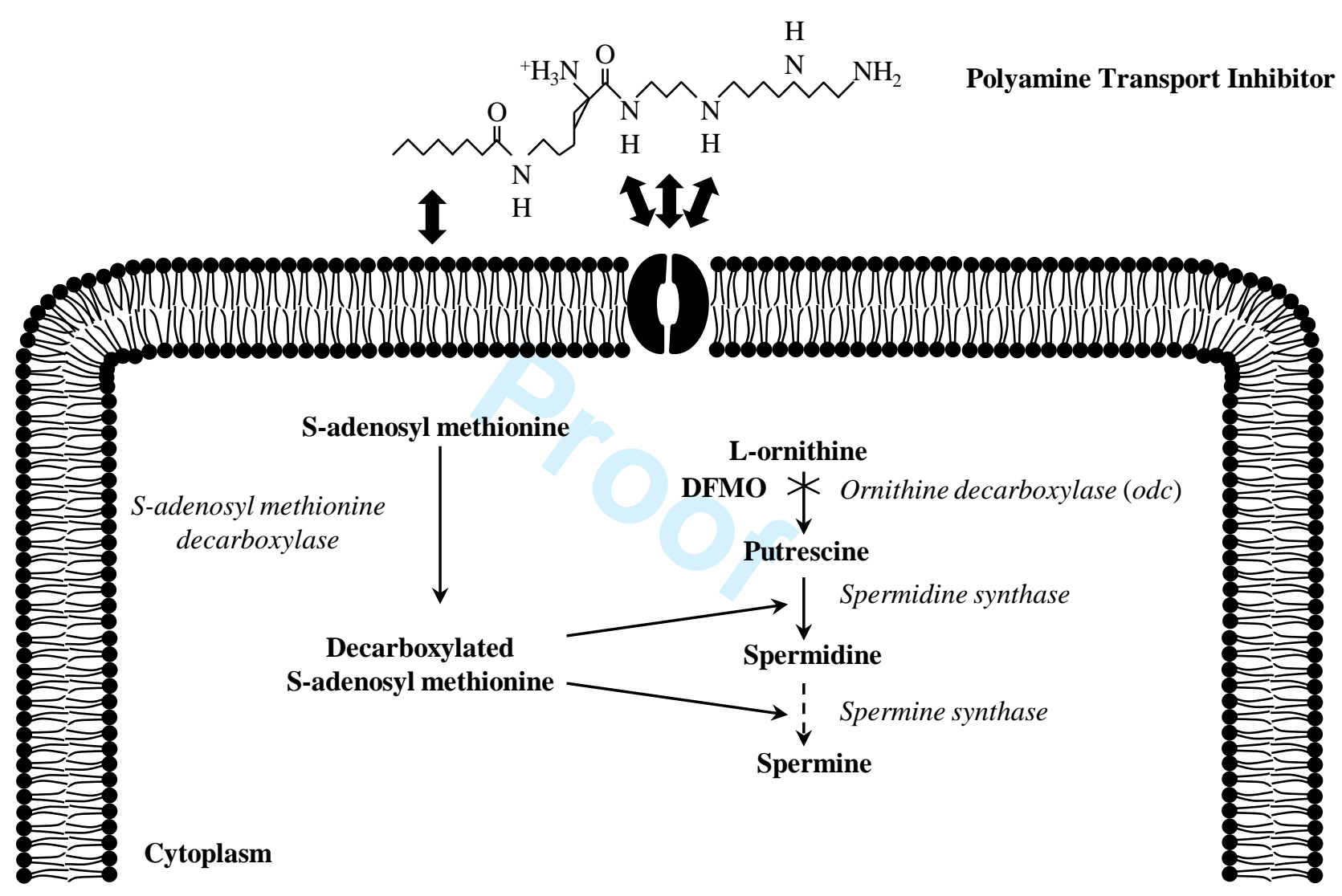




\section{Page 27 of 33}

A)

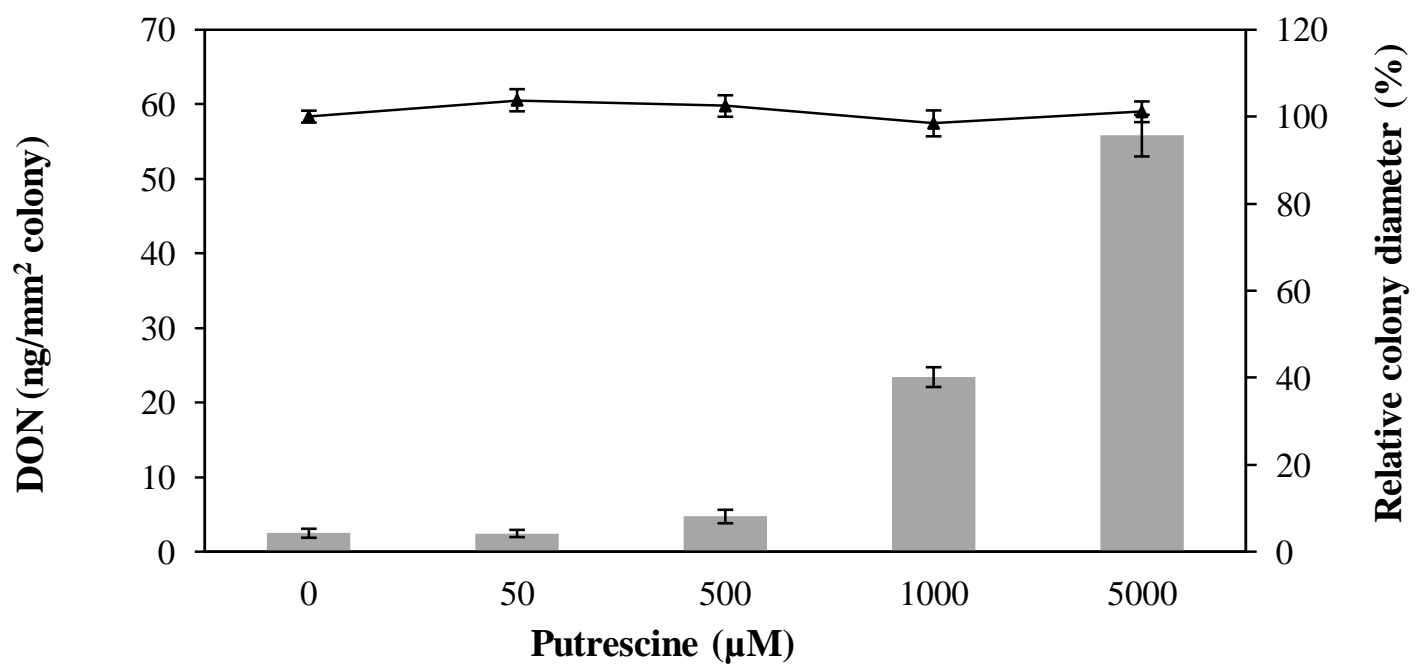

$\longrightarrow$ DON (ng/mm² colony) $\rightarrow$ Relative diameter (\%)

B)

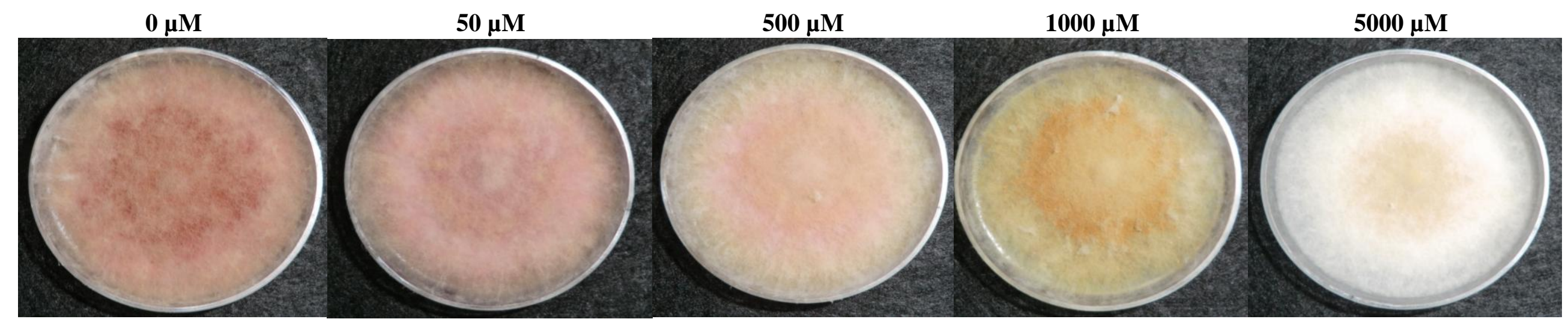




\section{Page 29 of 33}

A)

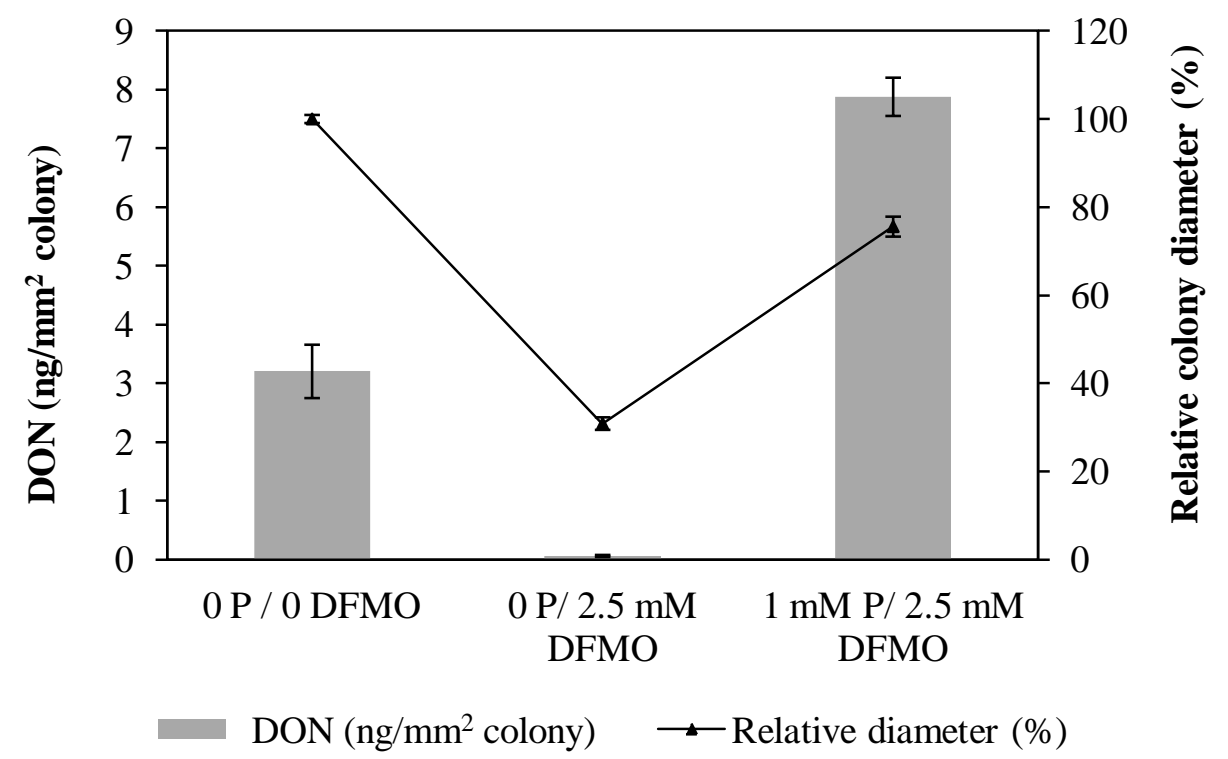

0 mM P/ O mM DFMO $\quad$ 0 mM P/ 2.5 mM DFMO 1 mM P/ 2.5 mM DFMO

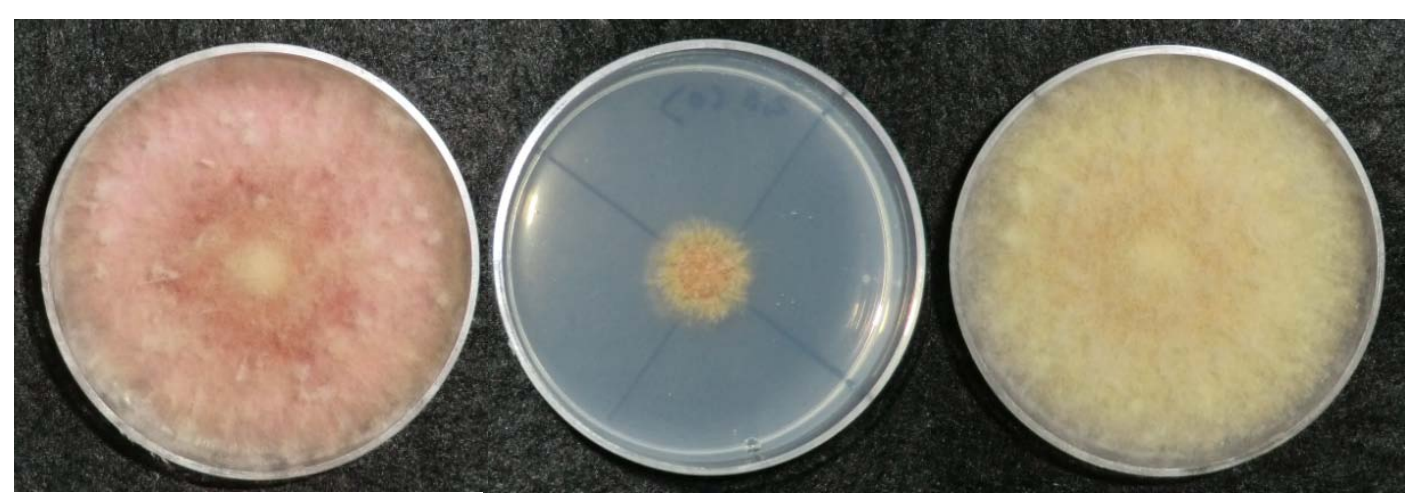


Page 30 of 33

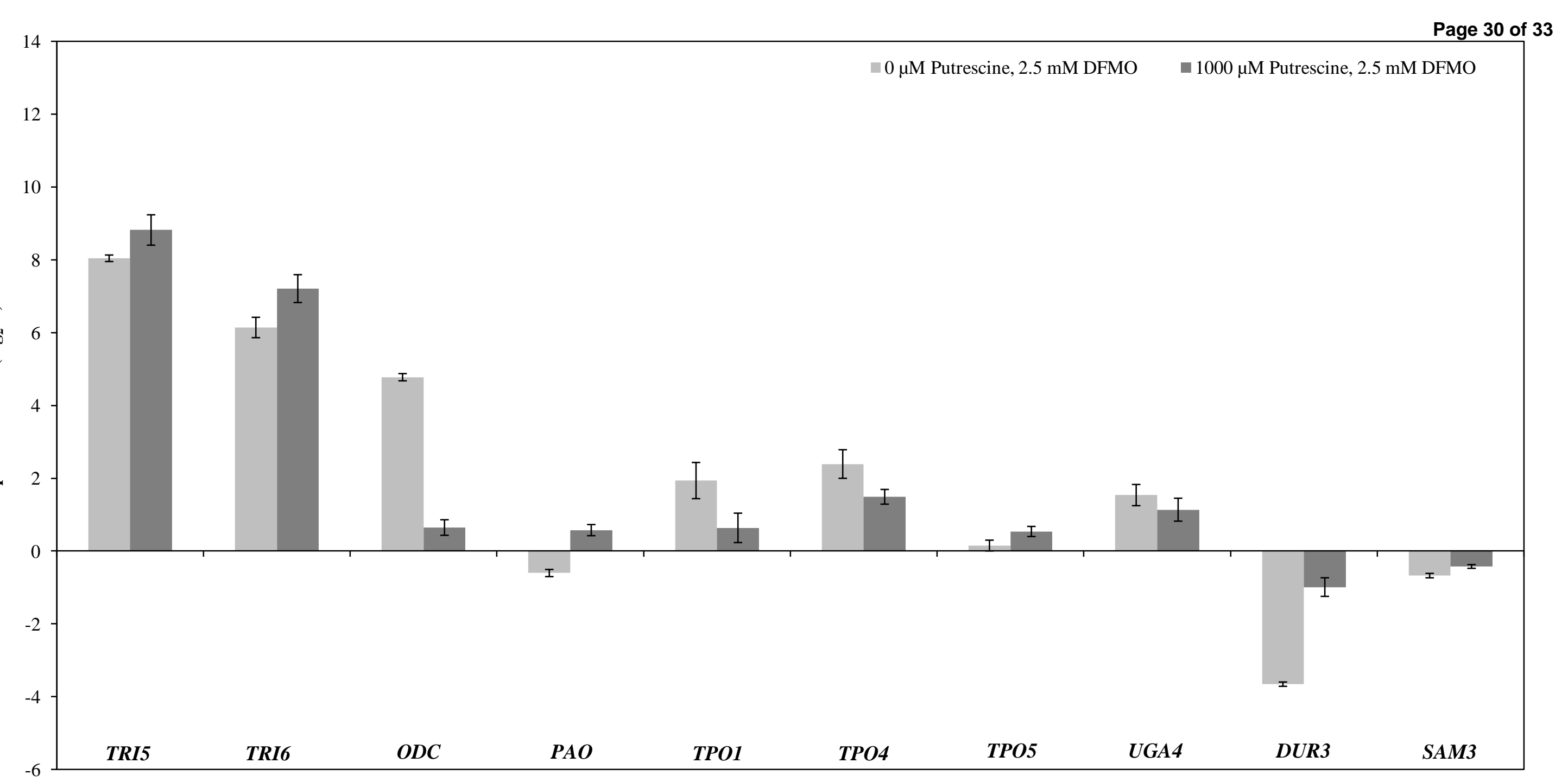




\section{Page 31 of 33}

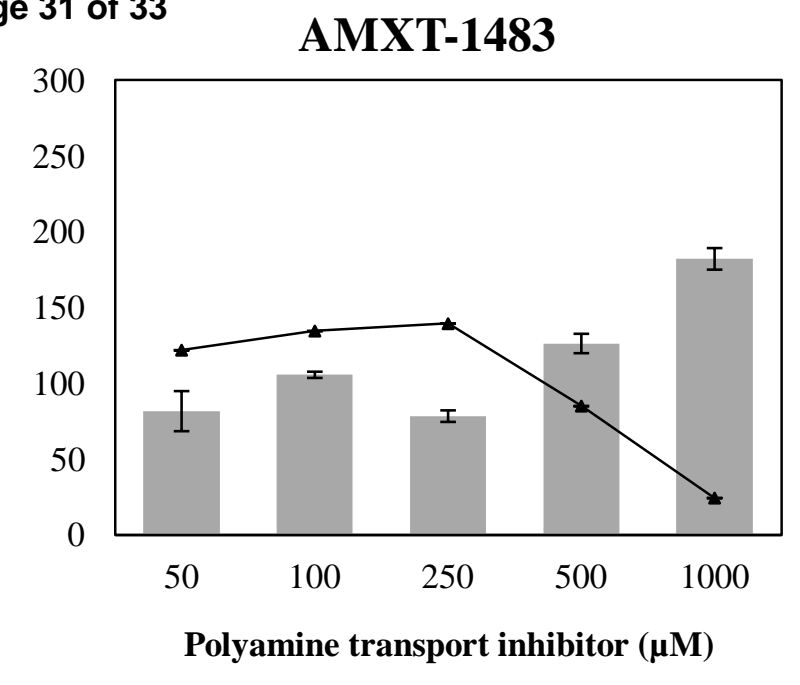

DON (\%) — colony diameter(\%)

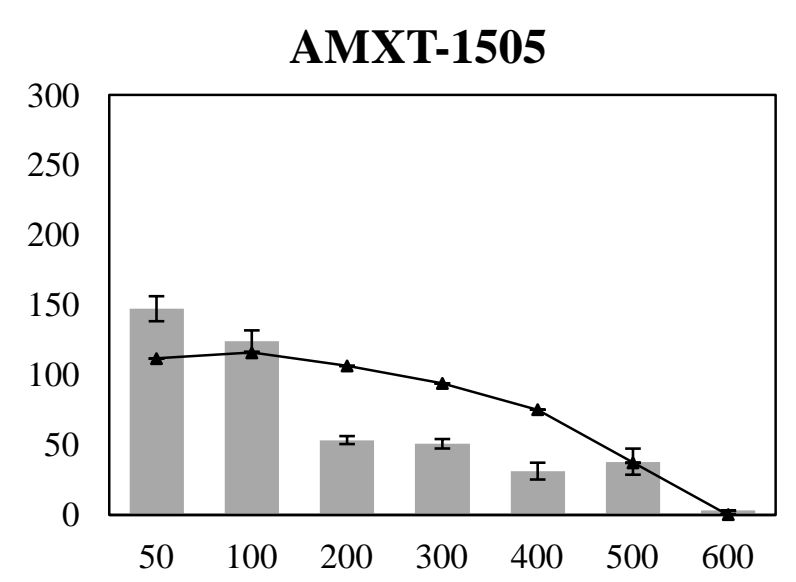

Polyamine transport inhibitor ( $\mu \mathrm{M})$

DON $(\%) \rightarrow$ colony diameter $(\%)$

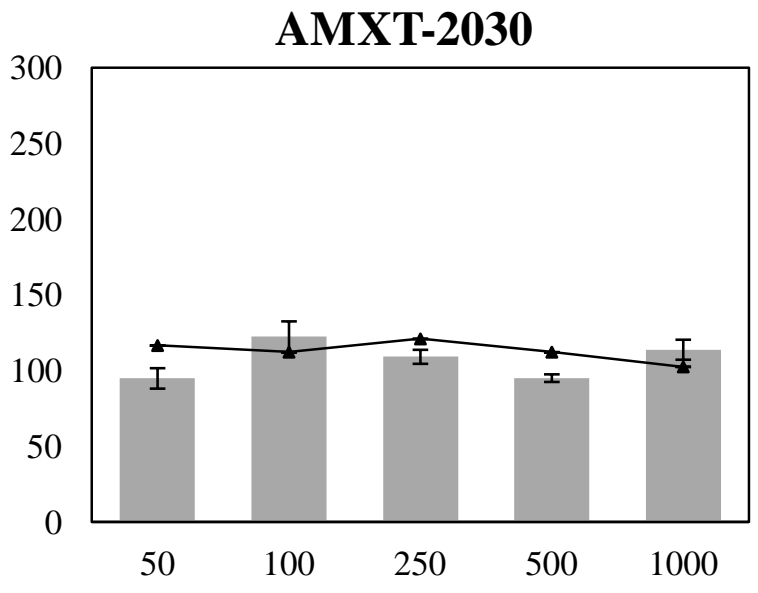

Polyamine transport inhibitor $(\mu \mathrm{M})$

DON $(\%) \longrightarrow$ colony diameter $(\%)$

\section{AMXT-3016}

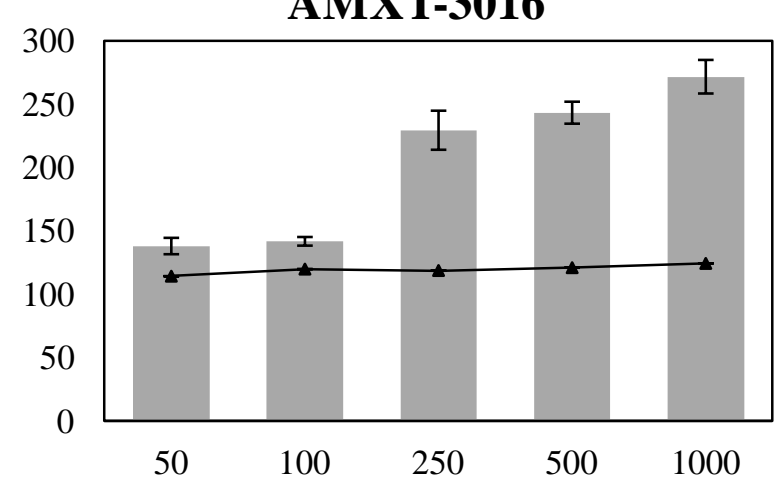

Polyamine transport inhibitor $(\boldsymbol{\mu M})$

DON (\%) — colony diameter (\%)

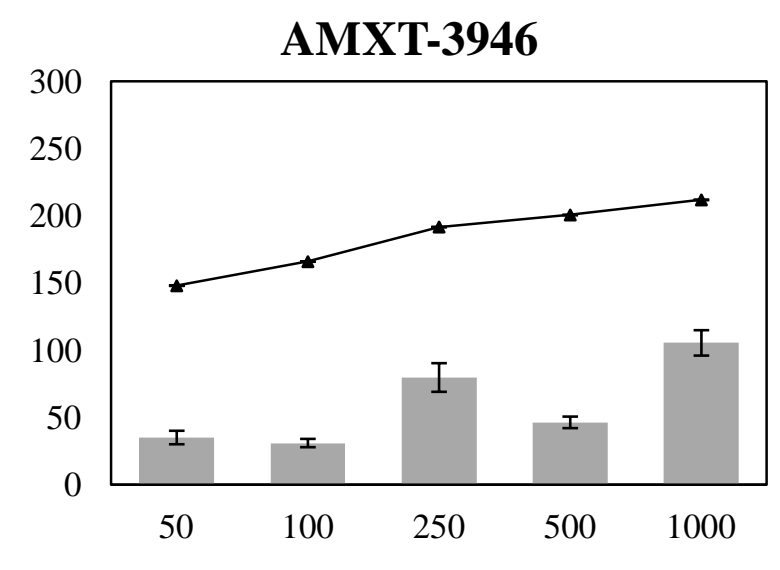

Polyamine transport inhibitor $(\boldsymbol{\mu M})$

DON (\%) $\rightarrow$ colony diameter(\%) 


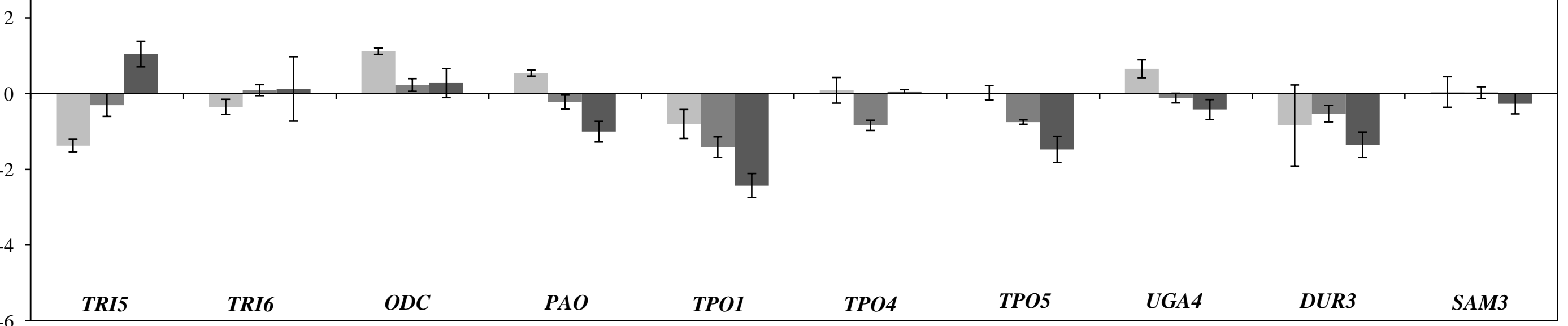

32

32
33 
Negative control
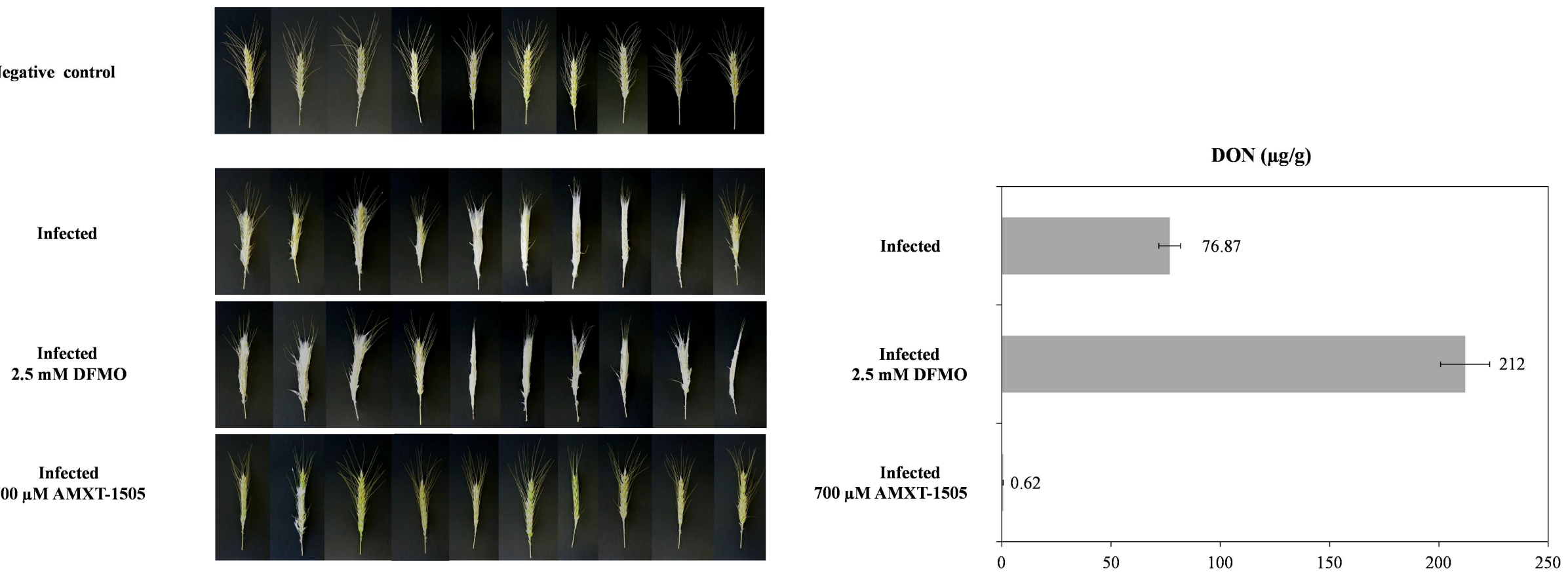